\title{
DISPERSIÓN URBANA Y SOSTENIBILIDAD AMBIENTAL EN LA COMUNITAT VALENCIANA
}

\author{
URBAN SPRAWL AND ENVIRONMENTAL SUSTAINABILITY IN VALENCIA \\ Salva Miñana-Fayos ${ }^{\mathrm{a},{ }^{,},}$, Eric Gielen ${ }^{\mathrm{b}}$, Gabriel Riutort-Mayol ${ }^{\mathrm{c}}$ \\ a Ingeniero en Geodesia y Cartografía, Licenciado en Geografía. salvaminyana@hotmail.com \\ ${ }^{\mathrm{b}}$ Departamento Urbanismo. Universitat Politècnica de Valencia. Camino vera S/N, 46022 Valencia. egielen@urb.upv.es \\ ${ }^{\mathrm{c}}$ Departamento de Ingeniería Cartográfica, Geodesia y Fotogrametría. Universitat Politècnica de Valencia. Camino vera S/N, 46022 \\ Valencia. gabriuma@upv.es
}

\begin{abstract}
:
The territory is configured by different urban models. During the last decades the tendency in many European countries has been forward to an urban sprawl development model. This kind of development causes several economic, social and environmental effects. This paper is focused on studying the environmental effects of the urban sprawl model for all municipalities of Valencia. These effects are measured over a set of 14 environmental indicators. First of all, an urban sprawl index is calculated by means of the Principal Component Analysis technique from the three more characteristic variables of the sprawl phenomenon. Finally, a regression model with spatially correlated effects is formulated with the aim of estimating the effects of the urban sprawl model on the environmental indicators. It is concluded that the sprawl model causes significant effects on most of the environmental indicators.
\end{abstract}

Key words: Urban Sprawl, Environmental Sustainability, Spatial Regression Model, Geographic Information System. Comunitat Valenciana

\section{Resumen:}

El territorio está configurado por distintos modelos urbanos y la tendencia en las últimas décadas y en muchas ciudades de Europa ha sido hacia un modelo de crecimiento disperso. Este tipo de crecimiento disperso ha llevado consigo fuertes cambios en los usos del suelo en algunas regiones, produciendo fuertes presiones y efectos territoriales, ambientales y socia-económicos considerables. El presente artículo estudia los efectos ambientales de este tipo de crecimiento urbano disperso en el conjunto de los municipios de la Comunitat Valenciana. En primer lugar, se elabora un índice que mide el nivel de dispersión urbana en cada municipio. Este índice de dispersión se obtiene mediante el cálculo del factor común (primera componente principal del Análisis de componentes Principales) a un conjunto de tres indicadores característicos del modelo urbano disperso: la densidad neta de viviendas, la compacidad del suelo urbano y la magnitud o extensión del suelo urbano en el municipio. En segundo lugar, se observa un conjunto de catorce indicadores de sostenibilidad ambiental relacionados con el metabolismo urbano, infraestructuras, zonas verdes y movilidad. Finalmente, se plantea un modelo estadístico de regresión con efectos correlados espacialmente con el objeto de determinar el efecto del nivel de dispersión urbana sobre los diferentes indicadores de sostenibilidad ambiental observados. Los resultados concluyen que el modelo urbano disperso genera efectos significativos sobre la mayoría de los indicadores ambientales elegidos.

Palabras clave: Dispersión Urbana, Sostenibilidad Ambiental, Modelo de Regresión Espacial, Sistema de Información Geográfica, Comunitat Valenciana

\section{Introducción}

El siglo XX, sobre todo en la segunda mitad, ha estado marcado por un acelerado proceso de urbanización en todo el planeta: el $80 \%$ de la población de España vive en áreas urbanas. Las ciudades se han ido organizando según distintos modelos urbanos. Cada región ha ido desarrollándose acorde a un modelo de crecimiento urbano distinto, y dando lugar a una ocupación del territorio diferente. En las últimas décadas, la tendencia ha sido hacia modelos de crecimientos dispersos 0 menos compactos. Esta expansión urbana dispersa, constituye uno de los factores que más ha influido en los cambios de uso del suelo en algunas regiones, produciendo fuertes presiones y efectos territoriales considerables. Este fenómeno de desarrollo urbano, se ha dado con gran intensidad en el arco mediterráneo a expensas de la agricultura y zonas de bosque.

La dispersión urbana es un fenómeno complejo, multidimensional y multiescala; una de sus características más relevantes es que el consumo de suelo crece más rápido que la población. Son muchas y diferentes las causas, principalmente de tipo social, demográfico y económico, que han dado lugar al aumento de este tipo de modelo urbano (Muñiz et al. 2006). Catalán et al. (2007) considera este fenómeno

‘Corresponding Author: Salva Miñana-Fayos, salvaminyana@hotmail.com 
como un proceso urbano relacionado con la madurez económica de las ciudades. Las causas pueden ser diversas en función del contexto histórico o geográfico de cada región, pero las consecuencias siempre son las mismas, produciendo una transformación de las características del territorio y creando nuevas estructuras y áreas funcionales modificando las actuales (Rueda 2002). Todo ello provoca una serie de efectos económicos, sociales y ambientales.

Por otro lado, el concepto de la sostenibilidad urbana se define como una nueva forma de gestión de las zonas urbanas. De forma general, la sostenibilidad urbana engloba tres aspectos diferenciados: cohesión social, sostenibilidad económica y territorial, y sostenibilidad ambiental (López 2013). En este trabajo nos centramos principalmente en el estudio de efectos relacionados con los aspectos ambientales de la sostenibilidad urbana, ya que éstos, en su gran mayoría, derivan del sistema urbano implantado en el territorio. Entre las consecuencias ambientales causadas por el modelo urbano disperso se pueden destacar: la destrucción de paisajes, el aumento del metabolismo urbano, la pérdida de suelo fértil, la impermeabilización del suelo, el aumento de la movilidad, el aumento del uso del coche y la pérdida de biodiversidad (Entrena 2005).

Las zonas urbanas son claves en el proceso de alteración de los equilibrios ambientales, de forma que analizar este proceso en relación al modelo urbano puede ayudar a conocer sus efectos y a plantear políticas territoriales que consigan unas ciudades más sostenibles.

\section{Objetivos}

El objetivo principal que se persigue en este estudio es analizar y cuantificar la relación que existe entre el nivel de dispersión urbana de los municipios de la Comunitat Valenciana y distintos indicadores de sostenibilidad ambiental, con el fin de conocer algunos de los efectos que tiene el modelo urbano disperso sobre el medio ambiente.

Para la consecución de este objetivo se plantean los siguientes objetivos específicos:

- Creación y cálculo de un índice de dispersión urbana para el conjunto de los municipios de la Comunitat Valenciana, a partir del análisis de una serie de variables características del modelo disperso.

- La medición y elaboración de indicadores municipales de sostenibilidad ambiental relacionados con los ámbitos de metabolismo urbano, infraestructuras, zonas verdes y movilidad.

- La estimación de los efectos que sobre los diferentes indicadores de sostenibilidad tiene el nivel de dispersión urbana de un municipio, a partir de la formulación de un modelo estadístico de regresión espacial.

\section{Metodología}

\subsection{Revisión bibliográfica sobre dispersión urbana y sostenibilidad ambiental}

La dispersión urbana afecta a todo el planeta, aún así no dispone de una definición universal. Se trata de un fenómeno complejo, ya que es multidimensional y multiescala (Zeng et al. 2014). La aproximación que suele dársele a la medición y caracterización del fenómeno de la dispersión urbana es a partir de la obtención de diferentes indicadores característicos, especialmente relacionados con aspectos morfológicos y demográficos de la ocupación del territorio. La densidad neta es el factor que aparece en la mayoría de trabajos sobre caracterización de la dispersión urbana (Angel et al. 2014; Frenkel and Ashkenazi, 2008; Colaninno et al. 2011; Gielen et al. 2017). La dispersión entre las manchas de suelo urbano es otro de los factores muy representativos de un modelo urbano disperso (Angel et al. 2014; Colaninno et al. 2011; Gielen et al. 2017). La fragmentación de usos en el espacio urbano es también representativo de un modelo disperso, por el contrario la mezcla de usos es más representativa de un modelo compacto (Frenkel and Ashkenazi 2008; Arribas-Bel et al. 2011).

Así mismo, la complejidad de la forma de la superficie abarcada por suelo urbano es también un factor característico de un modelo compacto, en cambio formas menos complejas y más regulares tienden a ser características de modelos más dispersos (Zeng et al. 2014; Colaninno et al. 2011). La ocupación del espacio libre, la concentración de edificios, así como la proporción de suelo urbano discontinuo y compacto dentro del suelo urbano, son también factores que se utilizan como medidas representantes del fenómeno de la dispersión (Gielen et al. 2017). Por último, la magnitud o proporción que abarca el suelo urbano en el territorio es un factor a tenerse en cuenta ya que es un factor importante en relación a las consecuencias derivadas de este tipo de modelos sobre el territorio (Gielen et al. 2017).

Por otro lado, las técnicas estadísticas multivariantes tradicionales como el análisis factorial y el análisis de componentes principales son las técnicas más comúnmente utilizadas con el objetivo de agregar y sintetizar en un único o varios factores la información contenida en todas estas variables relacionadas con el fenómeno de dispersión urbana (Mezzeti and Billari 2005; Frenkel and Ashkenazi, 2008).

En cuanto a la sostenibilidad ambiental, son muchas las entidades y autores que definen indicadores para medir y analizar la sostenibilidad urbana en todos sus ámbitos. El Observatorio de la Sostenibilidad en España emite un informe anual de Sostenibilidad por provincia evaluado sobre indicadores característicos de las distintas dimensiones de sostenibilidad urbana; indicadores socioeconómicos, ambientales y de sectores productivos, con el fin de conocer el nivel de sostenibilidad y así poder controlar el éxito de determinadas medidas o acciones que se van tomando. A menor escala, existe el Sistema Municipal de Indicadores de Sostenibilidad Urbana y Local creado por la Red de Redes de Desarrollo Local y Sostenible. Este sistema de indicadores abarca seis dimensiones; 
ocupación del suelo, complejidad urbana, movilidad sostenible, metabolismo urbano, cohesión social y biodiversidad. Se trata de indicadores homogéneos que permiten el seguimiento de la Estrategia Española de Sostenibilidad Urbana y Local y facilitan la comparación del grado de sostenibilidad de cualquier municipio de España.

Entre los efectos que el modelo de ciudad dispersa puede causar sobre los aspectos relacionados con el medioambiente se encuentran el aumento de la fragmentación del territorio afectando a la estabilidad ambiental (Rueda 2002), el aumento de los costes energéticos y el aumento del consumo de recursos (Rueda 2002; Entrena 2005; Muñiz et al. 2006). También, se han realizado estudios que demuestran un efecto significativo en la instalación de redes de abastecimiento y saneamiento de aguas, así como en el coste por habitante en alumbrado público (Bereciartúa 2006).

\subsection{Fuentes de información}

Para la obtención de las variables relacionadas con la dispersión urbana se han utilizado principalmente dos fuentes de información:

- La base de datos del Sistema de Información sobre Ocupación del Suelo de España (SIOSE), a partir de la cual se ha obtenido la superficie de suelo urbano mixto y urbano discontinuo pertenecientes al año 2011.

- El censo de población y viviendas del año 2011 (Instituto Nacional de Estadística), a partir del cual se ha obtenido el número de viviendas por municipio.

Por otro lado, para la obtención de los indicadores de sostenibilidad ambiental se han utilizado las siguientes fuentes de información:

- Encuesta de Infraestructuras y Equipamientos Locales (EIEL), gestionada por las diputaciones provinciales y de donde se han obtenido indicadores relacionados con el consumo de recursos y de infraestructuras: consumo de agua, volumen de residuos, puntos de luz, contenedores, superficie verde, red de tuberías y red de saneamiento.

- La base de datos espacial CartoCiudad, perteneciente al Instituto Geográfico Nacional (IGN), de donde se ha obtenido el indicador de viario urbano.

- Banco de datos territorial perteneciente al Instituto Valenciano de Estadística, de donde se ha obtenido el indicador de número de vehículos.

- Base de datos de la Entidad de Saneamiento de Aguas Residuales de la Comunitat Valenciana de la cual se ha obtenido el indicador de volumen de agua tratada.

- Base cartográfica 1:5000 del Instituto Cartográfico Valenciano, de donde se ha obtenido la superficie de piscinas.
- Censo de población y viviendas del IGN, de donde se han obtenido los indicadores relacionados con la movilidad, como el indicador de autocontención (número de personas ocupadas en el mismo municipio de residencia) y el indicador de número de personas que utilizan el vehículo para ir a trabajar.

- Catastro inmobiliario dependiente del Ministerio de Hacienda y Función Pública, a partir del cual se ha obtenido el número de alturas y viviendas para determinar el consumo energético por tipología de viviendas.

\section{3. Índice de dispersión urbana municipal}

En este trabajo para el cálculo del índice de dispersión urbana se han utilizado tres variables representantes de las características más importantes de la dispersión urbana: la densidad neta, la compacidad y la magnitud (Tabla 1). Estos indicadores recogen en gran medida el proceso de dispersión urbana. La densidad neta de edificación se obtiene como el número de viviendas dividido por la superficie de suelo urbano mixto. Para la obtención de la superficie de suelo urbano mixto se recurre a las coberturas de casco, ensanche y urbano discontinuo, recogidas en la base de datos del SIOSE. La compacidad vendrá definida como la proporción de superficie urbana discontinua que está ubicada más allá de la envolvente de 500 metros formada por las categorías de centro histórico y ensanche, en relación a la superficie urbana mixta total del municipio. Se ha utilizado un área de influencia de 500 metros para este cálculo, ya que se trata de una medida definida en la directriz 85, punto 2 de la Estrategia Territorial de la Comunitat Valenciana de 2011 para el cálculo de la superficie computable a efectos de la obtención de índices de ocupación de suelo para uso residencial. Finalmente, la magnitud se obtiene como la relación entre la superficie de suelo urbano mixto y la superficie total del término municipal.

Tabla 1: Indicadores para calcular el índice de dispersión urbana. Viv: $N^{\circ}$ viviendas por municipio. STM: Superficie término municipal. SUM: Superficie suelo urbano mixto. SUD

Superficie suelo urbano discontinuo. C: Casco urbano. e: Ensanche

\begin{tabular}{c|c} 
Indicador & Fórmula \\
\hline Densidad neta (Viv/ha) & $\frac{\text { Viv }}{S U M}$ \\
Compacidad (\%) & $\frac{S U D>500 \text { c.e }}{S U M}$ \\
Magnitud (\%) & $\frac{S U M}{S T M}$
\end{tabular}

El índice de dispersión urbana se obtendrá como el factor común a los tres indicadores, densidad neta, compacidad y magnitud, definidos anteriormente. El factor común de los tres indicadores se obtiene como la combinación lineal de los mismos, que estadísticamente maximiza la variabilidad. Éste se corresponde con la primera componente principal resultante de aplicar el método de Análisis de Componentes Principales (ACP), puesto que la primera componente principal es un consenso de la máxima varianza compartida entre todas 
las variables. El método ACP se basa en la descomposición en vectores y valores propios de la matriz varianzas-covarianzas del conjunto de los tres indicadores iniciales (Abdi and Williams, 2010). El método descompone la matriz $X$ de variables originales en función de las matrices $S$ y $A$ (Ec. 1), de forma que la matriz $S$ contiene las nuevas variables o componentes principales y la matriz $A$ los pesos o contribuciones de la combinación lineal.

$X=S \cdot A$

Para aplicar el método ACP se ha utilizado la librería FactoMineR (Husson et al. 2007) del software estadístico R (R Core Team 2016). El método de ACP se compone de un modelo lineal, y presupone normalidad en las variables de entrada. En nuestro caso, las variables densidad neta, compacidad y magnitud son claramente asimétricas, concentrándose gran cantidad de observaciones en valores bajos (Fig. 1). Para obtener más contraste entres estos valores bajos y, a su vez, obtener variables con distribuciones más normales, es conveniente aplicar alguna transformación a los datos. A las variables densidad neta y compacidad se les ha aplicado la transformación de la raíz cuadrada, y a la variable magnitud una transformación logarítmica. Ambas son transformaciones monótonas que no cambian el orden de los datos (Fig. 2).
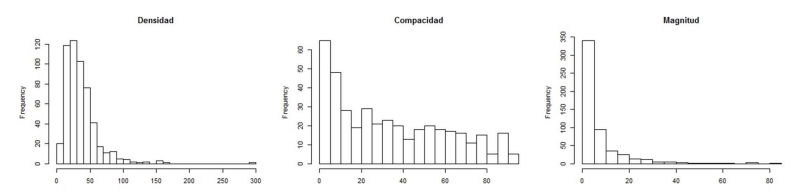

Figura 1: Histogramas sin transformar de las variables de dispersión urbana.
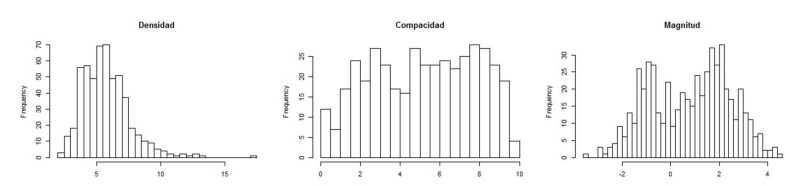

Figura 2: Histogramas transformados de las variables de dispersión urbana.

\subsection{Indicadores de sostenibilidad ambiental}

Para la caracterización de la sostenibilidad ambiental se han elaborado una serie de indicadores ambientales a nivel municipal. Los indicadores están calculados en función del número de viviendas, puesto que su cálculo respecto a la población conlleva una serie de problemas intrínsecos a la hora de reflejar el dato real, sobre todo en el caso de las segundas residencias o de la población vinculada residente pero no empadronada.

La elección de los indicadores se realiza en base a varios factores: posibilidad de cálculo a nivel municipal, viabilidad de la información, relevancia en el ámbito de la sostenibilidad ambiental y posibilidad de realizar un seguimiento temporal para poder medir el progreso. Algunos de los indicadores se han calculado directamente a partir del dato proporcionado por las fuentes de información, es el caso de los indicadores: consumo de agua, volumen de residuos, autocontención (número de personas ocupadas en el mismo municipio de residencia), personas que utilizan el vehículo para ir a trabajar, número de vehículos, número de puntos de luz, número de contenedores y longitud de la red de tuberías y de saneamiento. En otros casos, el indicador se ha obtenido a partir de operaciones de geoprocesamiento integradas en Sistemas de Información Geográfica: es el caso del indicador de superficie de piscinas que se ha obtenido a partir de la intersección espacial de la capa vectorial de piscinas del Instituto Cartográfico Valenciano y la capa vectorial de municipios del IGN. De la misma forma, el indicador de superficie verde se ha obtenido mediante la intersección de la capa vectorial de municipios del IGN y la cobertura de superficie verde de la capa de usos del suelo del SIOSE. El indicador de longitud vial urbano se ha obtenido a partir de la intersección de la capa vectorial de vial urbano de la base de datos CartoCiudad y la capa vectorial de municipios del IGN. Para el cálculo del indicador de volumen de aguas residuales tratadas, se ha realizado un reparto proporcional del caudal por población puesto que el dato existe a nivel de Estación Depuradora de Aguas Residuales y no a nivel municipal. Finalmente, para el cálculo del consumo de energía se ha realizado una estimación a partir de valores tipo dependientes de la tipología de residencia (vivienda unifamiliar y vivienda en bloque) y la zona climática (Tabla 2). La Tabla 3 muestra una relación de todos los indicadores ambientales finalmente observados.

Tabla 2: Consumo energético en Gigajulios (GJ) según zonas climáticas y tipos de viviendas. Fuente: Análisis del consumo energético del sector residencial en España. 2011. Instituto para la Diversificación y Ahorro de la Energía (IDAE)

\begin{tabular}{c|c|c|c|c} 
Viviendas & Ud & $\begin{array}{c}\text { Atlántico } \\
\text { Norte }\end{array}$ & Continental & Mediterráneo \\
\hline En Bloque & $\begin{array}{c}\text { GJ/Año } \\
\text { Vivienda }\end{array}$ & 26,3 & 35,3 & 22,1 \\
\hline $\begin{array}{c}\text { GJ/Año } \\
\text { Vivienda }\end{array}$ & 54 & 70,8 & 47,7
\end{tabular}

\subsection{Efecto de la dispersión urbana sobre los indicadores de sostenibilidad ambiental}

Se formula un modelo estadístico de regresión para cada una de las variables de sostenibilidad, con el fin de obtener su grado de correlación con el índice de dispersión urbana calculado en cada municipio. La formulación del modelo se realiza mediante la metodología de modelización jerárquica Bayesiana (Ntzoufras 2009). Un modelo estadístico de regresión se caracteriza por la definición de una distribución de probabilidad para la variable dependiente, denominada función de verosimilitud, y un regresor que define la relación funcional entre las variables explicativas y la variable dependiente. Se han considerado distribuciones normales para todas las variables independientes $Y_{j}$,:

$$
Y_{j} \sim \operatorname{Normal}\left(\mu_{j}, \sigma_{j}^{2}\right), \quad j=1, \text { J variables... }
$$

El regresor funcional entre la media $\mu_{j}$ y los factores explicativos considerados, se compone por la suma de dos componentes, una componente que es una función lineal de la covariable índice de dispersión urbana y otra componente que es un efecto aleatorio de estructura espacial (Ec. 2). El efecto aleatorio de estructura espacial recoge el efecto de que los municipios vecinos 
puedan tender a parecerse, en forma de suavizado local (Clayton et al. 1993):

$\mu_{j}(i)=\beta_{0, j}+\beta_{1, j} \cdot \operatorname{Sprawl}_{j}(i)+s p_{j}(i)$

Dónde:

$\mu_{j}(i)$ es la media de la función de verosimilitud de la variable dependiente $j$ en el municipio $i$.

$\beta_{0, j}$ es la media general del modelo para la variable $j$.

$\beta_{1, j}$ es el efecto medio del factor explicativo índice de dispersión urbana para la variable $j$.

$s p_{j}(i)$ es el efecto espacial de suavizado local en municipio $i$.

Tabla 3: Relación de indicadores de sostenibilidad ambiental.

\begin{tabular}{|c|c|c|}
\hline Nombre & Descripción & Unidades \\
\hline Pisci_v & Superficie piscinas & $\mathrm{m}^{2} /$ Vivienda \\
\hline H2O_v & Consumo agua & $\begin{array}{l}\text { Litros x Día / } \\
\text { Vivienda }\end{array}$ \\
\hline Edar_v & Vol. aguas residuales & $\begin{array}{l}\text { Litros x Día / } \\
\text { Vivienda }\end{array}$ \\
\hline Resid_v & Volumen residuos & $\begin{array}{l}\text { Kg } \times \text { Año / } \\
\text { Vivienda }\end{array}$ \\
\hline Cons_Elect & Consumo eléctrico & $\begin{array}{c}\text { Gigajulios x Año / } \\
\text { Vivienda }\end{array}$ \\
\hline Jard_v & Superficie verde & $\mathrm{m}^{2} /$ Vivienda \\
\hline Autocont & Autocontención & $(\%)$ \\
\hline TraconAuto & $\begin{array}{l}\text { Personas utilizan } \\
\text { vehículo para ir a } \\
\text { trabajar }\end{array}$ & $(\%)$ \\
\hline Vial_v & Longitud vial urbano & Ml / Vivienda \\
\hline Vehi_v & Parque vehículos & $\begin{array}{l}\text { No vehículos / } \\
\text { Vivienda }\end{array}$ \\
\hline Ptsluz_v & Puntos de luz & $\begin{array}{l}\text { Ptos. Luz / } \\
\text { Vivienda }\end{array}$ \\
\hline Conte_v & Contenedores & $\begin{array}{c}\text { (Conten. / } \\
\text { Vivienda) }{ }^{*} 10\end{array}$ \\
\hline Red_v & Red tuberías & $\begin{array}{l}\text { Ml Tubería / } \\
\text { Vivienda }\end{array}$ \\
\hline Sanea_v & Red saneamiento & $\begin{array}{l}\text { MI Saneamiento / } \\
\text { Vivienda }\end{array}$ \\
\hline
\end{tabular}

La elección de un modelo estadístico con componente espacial está motivada debido a que las variables dependientes (variables de sostenibilidad ambiental) presentan un marcado carácter espacial con posibles correlaciones debido a posiciones espaciales cercanas. Este efecto espacialmente estructurado se define mediante la distribución intrinsic conditional autorregresive (CAR) (Besag et al. 1991, Besag and Kooperberg, 1995). Esta distribución implica asociación espacial subyacente entre los valores de la variable sostenibilidad ambiental para un municipio $i$ y el de sus vecinos. Para el desarrollo y aplicación del modelo estadístico de regresión espacial, se ha usado el software estadístico $\mathrm{R}$ para el tratamiento y gestión de los datos, y el software WinBUGS (Lunn et al. 2000) para la modelización Bayesiana y estimación por un método de simulación basado en el método de Markov Chains Monte Carlo (MCMC) (Geman et al. 1984).

Para la selección y adecuación del modelo espacial planteado frente a la adopción de otros modelos más simples, se realiza una comparación entre el modelo espacial, el modelo donde solo se contempla el efecto lineal $\beta_{1}$ de la covariable Sprawl y el modelo nulo que solo contempla la constante del modelo. Para medir la capacidad explicativa de los tres modelos y seleccionar el mejor, se usa el estadístico Deviance y DIC (Deviance Information Criterion) (Spiegelhalter et al. 2002). Por otro lado, la diagnosis y validación del modelo se lleva a cabo mediante el análisis de la normalidad de los residuos del ajuste.

\section{Resultados}

\subsection{Cálculo del índice de dispersión urbana}

El índice de dispersión urbana se corresponde con la primera componente del ACP llevado a cabo sobre los tres indicadores iniciales: densidad neta, compacidad y magnitud. La Tabla 4a muestra las contribuciones de cada una de las variables iniciales sobre las nuevas componentes, resultado de aplicar el ACP. La primera componente está formada principalmente por las variables densidad neta y compacidad con contribuciones de 0,77 y 0,84 respectivamente y la variable magnitud con una aportación de 0,41. La segunda componente tiene la aportación principalmente de la variable magnitud con un 0,89 seguida de la variable densidad neta con un 0,40. La tercera dimensión es una dimensión más residual, correspondiéndose con un $18 \%$ del total de variabilidad y formada principalmente por la contribución de las variables densidad neta y compacidad (Tabla 4b). La primera dimensión explica un $50 \%$ del total de variabilidad y la segunda dimensión un $30 \%$.

Tabla 4: Resultados del método ACP. a) Pesos de los indicadores en cada dimensión. b) Porcentaje de varianza.

a)

\begin{tabular}{c|c|c|c} 
Componentes & Dim.1 & Dim.2 & Dim.3 \\
\hline Densidad neta & 0.77 & 0.40 & 0.48 \\
Compacidad & 0.84 & 0.07 & 0.53 \\
Magnitud & 0.41 & 0.89 & 0.17
\end{tabular}

b)

\begin{tabular}{c|c|c|c} 
Componentes & $\begin{array}{c}\text { Valores } \\
\text { Propios }\end{array}$ & $\%$ Varianza & $\begin{array}{c}\% \text { Varianza } \\
\text { Acumulada }\end{array}$ \\
\hline Dim 1 & 1,48 & 49,58 & 49,58 \\
Dim 2 & 0,96 & 32,05 & 81,64 \\
Dim 3 & 0,55 & 18,35 & 100,00
\end{tabular}

La Figura 3 muestra la distribución sobre el conjunto de municipios de la Comunitat Valenciana de los valores del índice de dispersión urbana obtenido, clasificado en cinco clases para su representación en el gráfico. La definición de los intervalos de clase se corresponde con los cuantiles $0 \%, 20 \%, 40 \%, 60 \%, 80 \%$ y $100 \%$ de la distribución de frecuencias del índice (Fig. 4). Este índice de dispersión urbana oscila entre $-3,53$ y 2,89 , y 
representa la variación de la compacidad a la dispersión, respectivamente. Los valores negativos se corresponden con modelos urbanos más compactos, mientras que los valores positivos muestran modelos urbanos más dispersos. Los municipios de la Comunitat Valenciana con valor negativo en el índice de dispersión urbana suman un total de 301 , y los municipios con índice de dispersión urbana positivo ascienden a 241. En la figura 5, se muestra por una parte, los municipios con valor negativo en el índice de dispersión calculado, y estos recogen un $42 \%$ de la población total en tan sólo un $21 \%$ de la superficie urbana mixta total. Por otra parte, los municipios con valor positivo en el índice de dispersión, representan un $58 \%$ de la población total de la Comunitat Valenciana y para ello ocupan un $79 \%$ de la superficie urbana mixta total. Estos datos evidencian claramente, el alto consumo de suelo por parte del crecimiento urbano disperso.

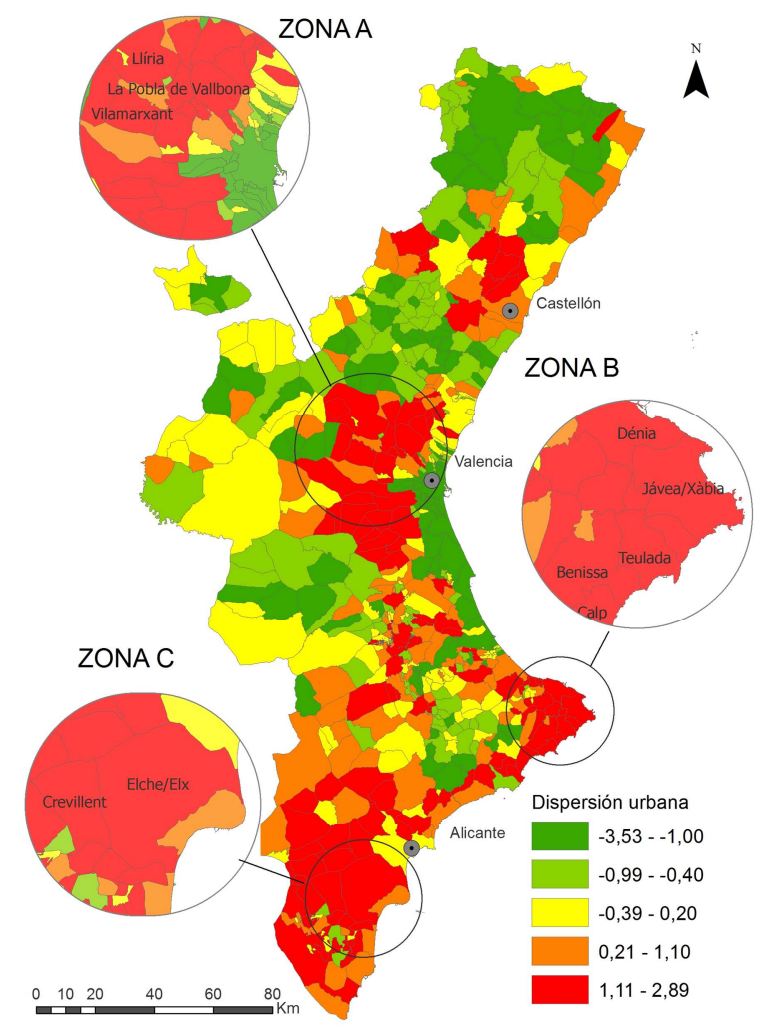

Figura 3: Índice de dispersión urbana de los municipios de la Comunitat Valenciana. Año 2011.

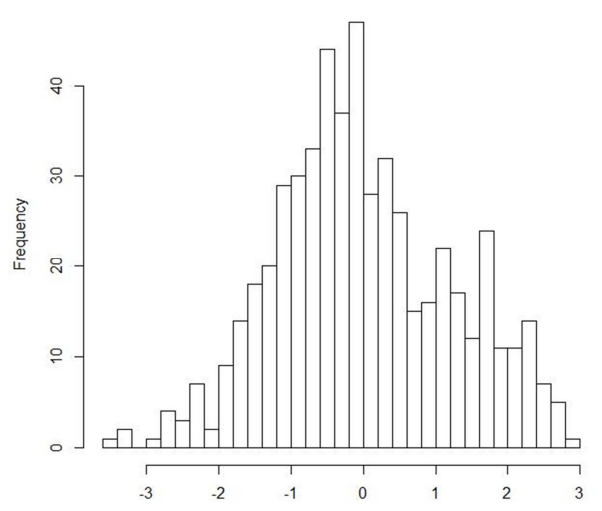

Figura 4: Histograma del índice de dispersión urbana calculado para los municipios de la Comunitat Valenciana.

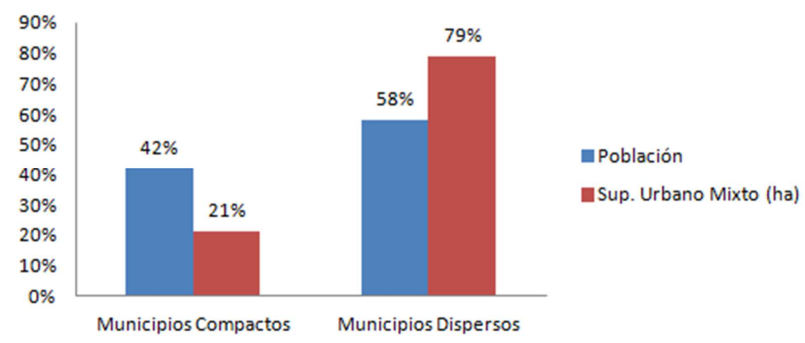

Figura 5: Comparación municipios dispersos y compactos.

\subsection{Efectos de la dispersión urbana sobre los indicadores de sostenibilidad ambiental}

Una vez calculado el índice de dispersión urbana por municipio y observados los indicadores de sostenibilidad ambiental, ya se está en consideración de calcular el efecto de la dispersión urbana sobre estos indicadores ambientales observados. De la aplicación del modelo de regresión espacial (Ec. 2), se obtienen las estimaciones de los parámetros del modelo (Tabla 5). El parámetro $\beta_{1}$ recoge el efecto lineal medio de la covariable índice de dispersión urbana sobre el indicador de sostenibilidad ambiental correspondiente. El parámetro $\sigma_{s p}^{2}$ es la varianza asociada al efecto de suavizado espacial, y el parámetro $\sigma_{Y}^{2}$ es la varianza residual. Las variables dependientes han sido previamente estandarizadas, por lo cual su variabilidad inicial es 1 . Se puede observar en la Tabla 5 como las varianzas residuales después del ajuste del modelo son bastante inferiores a 1, lo que indica cierta capacidad explicativa del modelo. La varianza estimada de efecto aleatorios $s p$ es significativa, lo que indica que se ha encontrado un efecto de suavizado espacial importante.

Tabla 5: Estimación de los parámetros del modelo de regresión espacial.

\begin{tabular}{c|c|c|c} 
Nombre & $\beta_{1}+$ error & $\sigma_{s p}^{2}$ & $\sigma_{Y}^{2}$ \\
\hline Pisci_v & $0,503 \pm 0,024$ & 0.84 & 0.17 \\
H20_v & $0,012 \pm 0,029$ & 0.59 & 0.46 \\
Edar_v & $-0,101 \pm 0,032$ & 1.16 & 0.42 \\
Resid_v & $0,086 \pm 0,037$ & 1.43 & 0.27 \\
Cons_Elect & $0,126 \pm 0,031$ & 0.93 & 0.40 \\
Jard_v & $0,584 \pm 0,026$ & 0.42 & 0.20 \\
Autocont & $-0,060 \pm 0,035$ & 0.72 & 0.46 \\
TraconAuto & $0,050 \pm 0,037$ & 1.11 & 0.25 \\
Vial_v & $0,352 \pm 0,031$ & 1.12 & 0.45 \\
Vehi_v & $0,021 \pm 0,024$ & 1.41 & 0.10 \\
Ptsluz_v & $0,035 \pm 0,033$ & 1.12 & 0.45 \\
Conte_v & $0,068 \pm 0,034$ & 1.03 & 0.40 \\
Red_v & $0,205 \pm 0,039$ & 0.86 & 0.42 \\
Sanea_v & $0,001 \pm 0,033$ & 0.93 & 0.50
\end{tabular}

Los efectos $\beta_{1}$ son efectos estandarizados, lo que permite, por un lado, su comparación y, por otro, su interpretación como efectos en proporción de tanto por uno. Los indicadores de sostenibilidad ambiental que más variación presentan al variar un punto la variable de dispersión urbana, son los indicadores de superficie de 
jardines por vivienda (Jard_v) con un efecto lineal medio del $58,4 \%$, y, por otra parte, el indicador de superficie de piscinas por vivienda (Pisci_v) con un efecto lineal medio de un $50,3 \%$. Además, se obtiene un $35,2 \%$ de efecto lineal medio sobre el indicador de longitud de vial por vivienda (Vial_v), un $20,5 \%$ de efecto lineal medio sobre el indicador de longitud de tuberías por vivienda (Red_v), y un efecto lineal medio del $12,6 \%$ sobre el indicador de consumo eléctrico por vivienda (Cons_Elect).

Presentan un efecto más bajo, los indicadores de puntos de luz por vivienda (Ptsluz_v) y contenedores por vivienda (Conte_v) con un $3 \%$ y $6,8 \%$ respectivamente. Destacar también con un efecto bajo, el indicador de residuos producidos por vivienda y año (Resid_v) con un $8,6 \%$ de efecto lineal medio y el indicador que recoge el porcentaje de gente que utiliza el coche para ir a trabajar (TraconAuto) con un $5 \%$ de efecto lineal medio producido por la variable de dispersión urbana.

Dos indicadores obtienen efectos negativos, lo que indica que disminuyen a medida que aumenta la dispersión urbana. Se trata del indicador de autocontención (Autocont) con un efecto del $-6 \%$ y del indicador de caudal de agua tratada por vivienda (Edar_v) con un $-10 \%$ de efecto lineal medio. Los indicadores de longitud de red de saneamiento por vivienda (Sanea v), consumo de agua por vivienda (H2O_v) y vehículos por vivienda (Nehi_v) no presentan ningún efecto significativo.

Los estadísticos Deviance y DIC obtenidos con el modelo espacial planteado, al ser significativamente menores a otros modelos más simples, un modelo sin el efecto de suavizado espacial y el modelo nulo (Tabla 6), índica que el modelo planteado es mejor modelo y se ajusta mejor a los datos.

Tabla 6: Estadísticos de bondad de ajuste Deviance y DIC para diferentes modelos

\begin{tabular}{c|c|c} 
& Deviance & DIC \\
\hline Modelo espacial & 4106 & 10277 \\
Modelo sin estructura espacial & 18288 & 18344 \\
Modelo Nulo & 19035 & 19076
\end{tabular}

\section{Discusión}

A la vista del índice de dispersión obtenido para el conjunto de los municipios de la Comunitat Valenciana (Fig. 3), se han podido detectar tres conjuntos de municipios destacados con una dispersión urbana elevada. Por una parte, cabe destacar el grupo de los municipios ubicados en el área metropolitana de Valencia (Fig. 3, zona A). Se trata de municipios ubicados en grandes vías de comunicación y por tanto con buena accesibilidad y próximos a grandes aglomeraciones de población. Estos municipios han absorbido parte de la población de Valencia por el proceso de periurbanización, convirtiéndose en ciudades dormitorio además de ofrecer residencias más tranquilas y en mayor contacto con la naturaleza, caso de Llíria, Villamarxant y Pobla de Vallbona. Este efecto de periurbanización se da también en los municipios del norte de la ciudad de Castellón y en los municipios de alrededor de Alicante. Por otro lado, el segundo grupo de municipios con valores muy altos de dispersión se corresponde con la zona de las comarcas de la Marina en la provincia de Alicante (Fig. 3, zona B). En estas comarcas se ha producido un incremento de suelo residencial de manera dispersa debido al turismo. Es el caso de municipios como Jávea, Dénia, Calp, Altea, Teulada, Benissa, entre otros. Por último, el tercer grupo se corresponde con municipios del Baix Vinalopó, Elx y Crevillent, en la provincia de Alicante (Fig. 3, zona C). Las causas del crecimiento disperso de este grupo se deben a la existencia de importantes ejes de comunicación como la autopista A-7 y autovía A-79 y a la aparición de tejido urbano discontinuo ligado al turismo.

Por otro lado, la elección de indicadores de sostenibilidad ambiental no ha estado exenta de limitaciones. La mayor limitación ha sido la dificultad para obtener datos ambientales fiables, actualizados y públicos a nivel municipal. Se ha detectado una falta de indicadores ambientales, sobre todo relacionados con el nivel de consumo de recursos, debido a que muchos servicios públicos que se dan en las zonas urbanas están externalizados a empresas adjudicatarias públicas de gestión privada, lo cual dificulta a menudo la obtención del dato, como es el caso del consumo de agua, generación de residuos, consumo de gas, electricidad y transporte; variables éstas muy importantes para determinar la sostenibilidad ambiental de una zona urbana. También, se han detectado diferencias en una misma variable proveniente de diferentes fuentes de información, caso del indicador de superficie de jardines (Jard_v) y vial urbano (Vial_v). Por último, comentar que muchos indicadores ambientales sólo existen a nivel municipal lo que limita el desarrollo de estudios a menor escala.

El modelo de regresión nos ha permitido cuantificar los efectos de la dispersión urbana sobre los diferentes indicadores de sostenibilidad ambiental elegidos. A pesar de que la variabilidad residual encontrada en el ajuste de algunas de las variables de sostenibilidad ha sido cercana al 50\% (Tabla 5), lo que indica que todavía queda una parte significativa de variabilidad que no ha sido recogida por el modelo para estas variables, los modelos formulados y ajustados son representativos y fidedignos ya que el modelo espacial planteado es mejor, en función de los estadísticos Deviance y DIC, que otros modelos (Tabla 6), la falta de independencia de los residos del ajuste de estas variables es muy ligera y los errores de estimación de los efectos de la variable explicativa son razonables y reducidos (Tabla 5).

Los indicadores con mayor efecto producido por el índice de dispersión urbana, son los indicadores de superficie de jardines (Jard_v), superficie de piscinas (Pisci_v), longitud de vial urbano (Vial_v) y red de tuberías de agua potable (Red_v) con un 58\%, 50\%, $35 \%$ y $20 \%$ respectivamente. Se trata de indicadores que representan infraestructuras que están claramente ligadas a las construcciones dispersas. En cuanto a indicadores de consumo, destacar los indicadores de consumo eléctrico (Cons_Elect) y generación de residuos (Resid_v) con un $13 \%$ y $9 \%$ respectivamente. Esto refleja el mayor consumo de energía en las zonas más dispersas, como se evidencia en el trabajo de Bereciartúa (2006). Para algunos de los indicadores de 
sostenibilidad elegidos, el efecto del índice de dispersión urbana no resulta significativo, como es el caso de los indicadores de consumo de agua (H2O_v), vehículos por vivienda (Vehi_v) y red de saneamiento (Sanea_V). Tanto en el indicador de consumo de agua como en el de red de saneamiento se esperaba a priori obtener un efecto significativo sin embargo no ha sido así. Cabe mencionar que ambos indicadores provienen de la base de datos EIEL y por tanto la inexistencia de efecto hace pensar en una baja calidad de los datos de estos indicadores.

Los efectos de los indicadores de autocontención (Autocont) y del indicador de gente que utiliza el coche para ir a trabajar (TraconAuto) presentan un efecto de $6 \%$ y $5 \%$ respectivamente. Se trata de efectos opuestos y evidencian, aunque de manera débil, el mayor uso del automóvil en los municipios más dispersos y el menor uso en los municipios más compactos. La movilidad es una variable difícil de calcular, teniendo que recurrir la mayoría de veces a encuestas personalizadas para obtener datos de calidad, lo cual hace pensar que no se ha podido recoger todo el efecto que la dispersión urbana produce sobre la movilidad.

Los indicadores de puntos de luz por vivienda (Ptsluz) y contenedores por vivienda (Conte_v) presentan un efecto bajo, con un $3 \%$ y $6,8 \%$ respectivamente. Esto puede ser debido, a que se trata de infraestructuras de servicios que atienden principalmente a criterios de población y que la dispersión urbana no influye tanto como la población.

Finalmente, destacar con un efecto en sentido negativo el indicador de caudal de agua tratada por vivienda (Edar_v) con un $-10 \%$ de efecto lineal medio, de tal forma que este indicador disminuye diez puntos porcentuales cuando aumenta un punto la dispersión urbana. Esto evidencia que en las zonas más dispersas no se está tratando toda el agua que se debería debido a que suponen mayores costes y que muchas zonas residenciales se consolidaron a mediados o finales del siglo $X X$, sin su correspondiente proceso de urbanización, por lo que muchas de ellas no se dotaron con una red de alcantarillado conectada a una Estación Depuradora de Aguas Residuales.

Con todo, en once de los catorce indicadores de sostenibilidad ambiental, ha sido posible establecer una relación directa con el nivel de dispersión urbana y cuantificar el efecto. Tan sólo en el caso de tres indicadores (H2O_v, Vehi_v, Sanea_v) no se ha encontrado efecto de la dispersión urbana.

\section{Conclusiones}

La principal dificultad de este tipo de estudios es la obtención del dato, ya sea por dificultad como por fiabilidad. Es necesaria la existencia de iniciativas políticas encaminadas a la recopilación de indicadores ambientales a nivel municipal, como la Agenda 21 que tenía como objeto alcanzar un desarrollo sostenible en el marco ambiental. Se ha tenido que recurrir hasta a ocho bases de datos oficiales diferentes para obtener únicamente catorce indicadores de sostenibilidad ambiental.

Es necesario establecer mecanismos que permitan calcular de manera eficiente y en la menor frecuencia posible, buenos indicadores de sostenibilidad ambiental de cada municipio o de las zonas urbanas.

Es interesante hacer notar que este estudio no pretende contraponer la ciudad dispersa frente a la ciudad compacta, tan solo trata de conocer los efectos de la dispersión urbana sobre el medio ambiente y cuantificarlos con el objeto de poder planificar modelos urbanos más sostenibles.

La consideración de una componente de suavizado espacial en el modelo de regresión planteado para obtener los efectos de la dispersión urbana sobre los indicadores de sostenibilidad ambiental, es un aspecto novedoso en este tipo de estudios, y que ha permito ajustar modelos más precisos para ajustar cada uno de los indicadores de sostenibilidad. Aún así, la variabilidad residual encontrada ha sido cercana al $50 \%$ en algunas de las variables de sostenibilidad. En un futuro trabajo se pretende probar la inclusión de un efecto aleatorio por municipio para intentar modelizar parte de esta variabilidad residual y mejorar incluso más la capacidad explicativa del modelo.

La obtención de los efectos de la dispersión urbana sobre la sostenibilidad ambiental, puede ser una herramienta de planificación urbanística, permitiendo evaluar el modelo urbano futuro planteado por cada municipio en su Plan General de Ordenación Urbana. A partir de esta herramienta se podría simular el crecimiento de las nuevas zonas urbanizadas, recalcular el nivel de dispersión y de esta manera, prever el efecto ambiental resultante.

\section{References}

ABDI, H. and WILLIAMS, J., 2010. Principal component analysis. WIREs Computational Statistics 2(4), pp.433-459, doi:10.1002/wics.101.

ANGEL, S., PARENT, J. and CIVCO, D., 2007. Urban Sprawl metrics: An analysis of global urban Expansion using GIS. ASPRS 2007 Annual Conference, Tampa, Florida. May 7-11, 2007.

ARRIBAS-BEL D., NIJKAMPY, P. and SCHOLTEN, H., 2011. Multidimensional urban sprawl in Europe: a self-organizing map approach. Computers, Environment and Urban Systems 35(4), pp. 263-275.

BERECIARTÚA, P.L., 2006. El crecimiento en baja densidad en Bizkaia. Urban Sprawl. XVI Congreso de Estudios Vascos: Desarrollo Sostenible-IT. En futuro. Donostia: 2006, pp.147-156.-ISBN-10: 84-8419-022-6; ISBN-13: 978-848419-022-6.

BESAG, J.E., YORK, J.C. and MOLLIÉ, A., 1991. Bayesian image restoration with two applications in spatial statistics with discussion. Annals of the Institute of Statistical Mathematics, 43, pp.671-681.

BESAG, J.E. and KOOPERBERG, C., 1995. On conditional and intrinsic autoregressions. Biometrika, 82, pp.733-746. 
CATALAN, B., SAURI, D. and SERRA, P., 2008. Urban sprawl in the Mediterranean? Patterns of growth and change in the Barcelona Metropolitan Region 1993-2000. Landscape and Urban Planning. 2008, 85, pp. 174-184.

CLAYTON, D., BERNARDINELLI, L. and MONTOMOLI C., 1993. Spatial correlation in ecological analysis. International Journal of Epidemiology, 22, pp. 1193-1202.

COLANINNO, N., CERDA. J. and ROCA. J., 2011. Spatial patterns of land use: morphology and demography, in a dynamic evaluation of urban sprawl phenomena along the Spanish Mediterranean coast. " 51 st European Congress of the Regional Science Association International”, pp. 1-18.

ENTRENA, F., 2005. Procesos de periurbanización y cambios en los modelos de ciudad. Un estudio europeo de casos sobre sus causas y consecuencias. Papers: revista de sociología, $\mathrm{n}^{\circ} \mathbf{7 8}, \mathrm{pp}$. 59-88.

FRENKEL, A. and ASHKENAZI, M., 2008. Measuring urban sprawl: how can we deal with it? Environment and Planning B: Planning and Design. 2008, volume 35, 99 pp. 56-79. DOI: 10.1068/b32155.

GIELEN, E., RIUTORT-MAYOL, G., PALENCIA JIMÉNEZ, J.S. and CANTARINO-MARTÍ, I., 2017. An urban sprawI index based on multivariate and Bayesian factor analysis with application at the municipality level in Valencia. Environment And Planning B-Planning \& Design 1 - 27. DOI: 10.1177/2399808317690148.

GEMAN, S. and GEMAN, D., 1984. Stochastic relaxation, gibbs distributions, and the bayesian restoration of images. IEEE Trans Pattern Anal Mach Intell. 1984, 6(6), pp.721-41.

HUSSON, F., JOSSE, J., L`E, S. and MAZET, J., 2007. FactoMineR: Factor Analysis and Data Mining with R. R package version 1.04, URL http://CRAN.R-project.org/package=FactoMineR.

LÓPEZ, D.A., 2013. La sostenibilidad urbana en la Unión Europea. M+A. Revista electrónic@ de Medio Ambiente. 2013, Volumen 14, número 2, pp. 38-62.

LUNN, D.J., THOMAS, A., BEST, N., and SPIEGELHALTER, D., 2000. WinBUGS a Bayesian modelling framework: concepts, structure, and extensibility. Statistics and Computing, 10:325337.

MEZZETTI, M. and BILLARI, F.C., 2005. Bayesian correlated factor analysis of socio-demografic indicators. Stat Methods Appt. 14, pp. 223-241.

MUÑIZ, I., GARCÍA, M.A. and CALATAYUD, D., 2006. SPRAWL. Definición, causas y efectos. Volumen 6 de Document de treball / Universitat Autónoma de Barcelona. Departament d'economia Aplicada, 2006, 39 pages.

OBSERVATORIO DE LA SOSTENIBILIDAD EN ESPAÑA, 2011. Informe de sostenibilidad en España 2011. Observatorio de la sostenibilidad en España. Mundiprensa.

NTZOUFRAS, I., 2009. Bayesian Modeling using WinBUGS. Wiley, 520 pages.

R CORE TEAM, 2016. R: A language and environment for statistical computing. R Foundation for Statistical Computing, Vienna, Austria. URL https://www.R-project.org/.

RUEDA, S., 2002. Els costos ambientals dels models urbans dispersos. Papers. Regió Metropolitana de Barcelona, $n^{\circ}$ 36, maig 2002, pp. 73-104.

SPIEGELHALTER D.J., BEST N.G., CARLIN B.P. and VAN DER LINDE, A., 2002. Bayesian measures of model complexity and fit (with discussion). J. Roy. Statist. Soc. B. 64, pp. 583-640.

ZENG, C.H., SANWEI. H.E. and JIAXING. C., 2014. Multi-Level and Multi-Dimensional Measuring on Urban Sprawl: $A$ Case Study in Wuhan Metropolitan Area, Central China. Sustainability 2014, 6, pp. 3571-3598; DOI:103390/su6063571. 